\title{
Generalized Poisson and Geometric Distributions - An Alternative Approach
}

\author{
Md. Tareq Ferdous Khan \\ Department of Mathematical Sciences, Florida Atlantic University, Boca Raton, FL 33431, USA \\ mdtareqferdo2017@fau.edu \\ Mian Arif Shams Adnan \\ Department of Mathematical Science, Ball State University, Muncie, IN 47304, USA \\ maadnan@bsu.edu \\ Md. Forhad Hossain \\ Department of Statistics, Jahangirnagar University, Dhaka-1342, Bangladesh \\ forhad.ju88@yahoo.com \\ Abdullah Albalawi \\ Department of Mathematical Science, Ball State University, Muncie, IN 47304, USA \\ पReceived प00 DFK2016 \\ Accepted प0०-XQ 2010
}

\begin{abstract}
An alternative approach of Poisson and Geometric distributions having a more general form of sampling method are suggested in this paper and defined them as generalized Poisson and generalized Geometric distributions respectively. It is evident that the traditional forms of both the distributions are the special cases of the proposed generalized distributions. Moreover, some distributional properties of the suggested distributions are derived here. The study also fixes the assumptions under which generalized Binomial distribution reduces to generalized Poisson distribution along with proof.

Keywords: Generalized Poisson distribution; Generalized Geometric distribution; Sampling method; Distributional properties.
\end{abstract}

Mathematics Subject Classification: 60E05, 62E10, 62E15, 62E20.

\section{Introduction}

The Poisson and Geometric distributions and their applications in statistical modeling and in many other scientific fields are well recognized. The traditional Poisson variate corresponds to the number of occurrences of the rare event in a fixed interval of time or space or other intervals and assumed to lie in discrete order between 0 to $\infty$. On the other hand, the usual Geometric variate is used to present the number of failure preceding the first success having the same range of the values of Poisson variate. But, in real world the event may occur in different fashions. Consider the sampling scheme where the number of occurrences possess the sequence such as (i) $0,2,4, \cdots, \infty$, (ii) $2,4, \cdots, \infty$, (iii) $3,6,9, \cdots, \infty$, (iv) $1,4,7, \cdots, \infty$ and so on. For instance, consider the total number of births those who born as twins at a particular hospital during a specified time interval. Let us define the number of new births as success and thus the number of success possess values 
$0,2,4, \cdots, \infty$. The usual Poisson distribution is completely helpless to deals with this particular sequence of number of success along with others mentioned above. Again, consider the example of twin births in geometric sense. In the sampling scheme, let us define the event as success if both the twins are male and stopped the sampling and number of births as twins in either combination of girl and boy or both girls are considered as failure. With these sequences of the values of the random variable, the traditional Geometric distribution cannot be applied to find a certain probability of a particular event.

In order to deal with the problems where traditional distributions are unaided, our study have suggested new generalization of the traditional Poisson and Geometric distributions where the random variable for each distribution is expressed by an arithmetic progression $a(n-1) d$, where $a$ is a non-negative integer representing the minimum values of the random variable, $n$ is a pre-assigned non-negative integer indicating the number of trails and $d$ is also a positive integer representing the concentration of the occurrences.

A series of studies have carried out under the heading generalization of Poisson distribution where the key concept our work is completely different. Consul and Jain $^{1}$ first suggested generalization of Poisson distribution having two parameters $\lambda_{1}$ and $\lambda_{2}$ which is obtained as a limiting form of the generalized negative Binomial distribution. In usual Poisson distribution, the mean and variance are same, while the variance of the suggested generalized distribution is greater than, equal to or smaller than the mean depending on whether the value of the parameter $\lambda_{2}$ is positive, zero or negative. Later Consul ${ }^{2}$ studied more extensively the distribution to cover the diversity of the observed number of occurrences for various factors. He also mentioned that proving the sum of all of the probabilities to unity is very difficult. In this context, Lerner et al. ${ }^{3}$ provide a more direct proof using the analytic functions. Some remarks on generalized negative binomial and Poisson distributions were made by Nelson ${ }^{4}$. Paul ${ }^{5}$ proposed a generalized compound Poisson model for panel data analysis on consumer purchase. Lin $^{6}$, in his study discussed about the generalized Poisson models and their applications in insurance and finance sector. On the basis of gamma function and digamma function a new two-parameter count distribution is derived by Hagmark ${ }^{7}$. He unveiled that the derived distribution can attain any degree of over/under dispersion or zero-inflation/deflation where the usual Poisson model has no dispersion flexibility.

Several authors have worked on generalization of the Geometric distribution which are also differ from the concept of our current work. Mishra ${ }^{8}$ proposed generalized geometric series distribution (GGSD) and shown that traditional geometric and Jain and Consul's 9 generalized negative binomial distribution are the special cases of the proposed distribution. A generalized Geometric distribution and its properties was introduced and studied by Philippou et al. ${ }^{10}$ from the motivation of the work by Philippou and Muwaf ${ }^{11}$. The distribution was defined under the heading of Geometric distribution of order $k$ where the distribution turned into the traditional form if order $k=1$. Considering the length-biased version of the generalized log-series distributions by Kempton ${ }^{12}$ and Tripathi and Gupta ${ }^{13}$, Tripathi et al. ${ }^{14}$ derived two version of two parameter generalized Geometric distribution. Another generalization of the Geometric distribution having two parameters was obtained by Gomez-Deniz ${ }^{15}$ obtained. He showed the generalization can be obtained either by using Marshal and Olkin ${ }^{16}$ scheme and adding a parameter to the Geometric distribution or from generalized exponential distribution presented in the same paper by Marshal and Olkin. Nassar and Nada ${ }^{17}$ discovered a five parameter new generalization of the Pareto-geometric distribution by compounding Pareto and Geometric distribution and generalized it by logit of the beta random variable.

\subsection{Proposed Generalized Poisson Distribution}

In the count data model, the traditional Poisson variate representing the number of occurrences takes the value ranges from 0 to $\infty$. Consider the following sequences for the values of the Poisson variate indicating the number of occurrence:

(i) $0,2,4, \cdots, \infty$

(ii) $2,4, \cdots, \infty$

(iii) $3,6,9, \cdots, \infty$ 
(iv) $1,4,7, \cdots, \infty$

and so on.

The arithmetic progression $a+n d$ can be used to represent the number of occurrences where, $a$ is a nonnegative integer representing the minimum number of occurrence, $d$ is a positive integer representing the concentration of occurrence and $n$ is a non-negative integer indicating the total number of trails. To tackle with the situation where the number of occurrences follows an arithmetic progression, we formulate a probability function and defined as generalized Poisson distribution. The sequence can easily take the traditional sequence $0,1,2, \cdots, \infty$ of Poisson distribution for the values $a=0$ and $d=1$ and then our proposed distribution turned into the usual Poisson distribution. Thus, a series of probabilistic problems can be solved by the proposed distribution including the problems solved by the traditional one. In this section, we define the proposed distribution and provide some of its important properties.

Definition 2.1.1: A random variable $X$ is said to have a Poisson distribution with parameter $\lambda, a, n$ and $d$ if it has the following probability mass function

$$
P(x ; \lambda, a, n, d)=\frac{\lambda^{x}}{x ! \sum_{n=0}^{\infty} \frac{\lambda^{a+n d}}{(a+n d) !}} \quad ; x=a, a+d, a+2 d, \cdots, a+n d
$$

where, $a \geq 0$ is the minimum number of occurrence, $d>0$ is the concentration of occurrence, $n$ is a preassigned non-negative integer such that $a=0$ if $n=0$ and $\lambda \geq 0$ is the mean number of occurrences.

It can be clearly shown that $P(x ; \lambda, a, n, d) \geq 0$ and $\sum_{x=a}^{a+n d} P(x ; \lambda, a, n, d)=1$ for different values of $X$ in terms of $a \geq 0, d>0$ and $n>0$. Hence, the suggested function of generalized Poisson distribution is a probability mass function.

The generalized Poisson distribution tends to the traditional Poisson distribution when minimum number of occurrence $a=0$ and concentration of occurrence $d=1$. Thus, we may conclude that the traditional Poisson distribution is a special case of the proposed generalized Poisson distribution. The advantage is to give the solution of finding probabilities of the count data where the values of the random variable can take infinite number of sequence including the traditional $0,1,2, \cdots, \infty$.

\section{Theorem 2.1.1: Derivation of Generalized Poisson Distribution.}

Proof: We proposed generalized Binomial distribution in another paper where the number of success is represented by an arithmetic progression. The form of the probability mass function of generalized Binomial variate $X$ with parameter $a, n, d$, and $p$ is

$P(x ; a, n, d, p)=\frac{\left(\begin{array}{c}a+n d \\ x\end{array}\right) p^{x} q^{a+n d-x}}{\sum_{x=a}^{a+n d}\left(\begin{array}{c}a+n d \\ x\end{array}\right) p^{x} q^{a+n d-x}} \quad ; x=a, a+d, a+2 d, \cdots, a+n d$

Under the following assumptions, the generalized Poisson distribution can be derived from generalized Binomial distribution.

i) $p$, the probability of success in a Bernoulli trail is very small. i.e. $p \rightarrow 0$.

ii) $n$, the number of trails is very large. i.e. $n \rightarrow \infty$.

iii) $(a+n d) p=\lambda$ is finite constant, that is average number of success is finite. Under this condition, we have 


$$
(a+n d) p=\lambda \quad \therefore p=\frac{\lambda}{(a+n d)} \text { and } q=1-\frac{\lambda}{(a+n d)}
$$

The remaining part of the derivation is presented in Appendix.

Theorem 2.1.2: The moment generating function of the generalized Poisson distribution and its first four raw and central moments.

The moment generating function of the generalized Poisson distribution is

$$
M_{X}(t)=\frac{1}{\sum_{n=0}^{\infty} \frac{\lambda^{a+n d}}{(a+n d) !}} \sum_{n=0}^{\infty} \frac{\left(\lambda e^{t}\right)^{a+n d}}{(a+n d) !}
$$

Differentiating it with respect to $t$ in first to $4^{\text {th }}$ order and equating $t=0$, we obtain the first four raw moments respectively and which are as follows:

$$
\begin{aligned}
& \mu_{1}^{\prime}=\frac{\sum_{n=0}^{\infty}(a+n d) \frac{\lambda^{a+n d}}{(a+n d) !}}{\sum_{n=0}^{\infty} \frac{\lambda^{a+n d}}{(a+n d) !}} \\
& \mu_{2}^{\prime}=\frac{\sum_{n=0}^{\infty}\{a+n d\}^{2} \frac{\lambda^{a+n d}}{(a+n d) !}}{\sum_{n=0}^{\infty} \frac{\lambda^{a+n d}}{(a+n d) !}} \\
& \mu_{3}^{\prime}=\frac{\sum_{n=0}^{\infty}(a+n d)^{3} \frac{\lambda^{a+n d}}{(a+n d) !}}{\sum_{n=0}^{\infty} \frac{\lambda^{a+n d}}{(a+n d) !}} \\
& \mu_{4}^{\prime}=\frac{\sum_{n=0}^{\infty}(a+n d)^{4} \frac{\lambda^{a+n d}}{(a+n d) !}}{\sum_{n=0}^{\infty} \frac{\lambda^{a+n d}}{(a+n d) !}}
\end{aligned}
$$

The corresponding central moments can be obtained by using the relationship between the raw and central moments. Consequently, we may find the measures of skewness and kurtosis. The form of the central moments and thus measures of skewness and kurtosis are seem to long and complicated equations and not in a concrete form, but very much simple to calculate the mentioned properties for specific values of $a$ and $d$.

All of the raw moments, central moments and measures of skewness and kurtosis derived from the generalized Poisson distribution tend to the form of traditional Poisson distribution when $a=0$ and $d=1$, which again justify that property that the usual Poisson distribution is the special case of the generalized Poisson distribution.

\subsection{Proposed Generalized Geometric Distribution}

In this section, we discuss about the generalized Geometric variate having relatively more general form of the values represented by an arithmetic progression which is mentioned above in the generalized Poisson 
distribution case and its distribution and the properties. We have proposed the distribution for the random variable taking values in the form $a+n d$ where $a$ is a non-negative integer representing minimum number of failure, $d$ is a positive integer indicating how the failures are occur, that is concentration of occurrence of failure per trail and $n>0$ is an integer representing the total number of trails. Clearly, the range of the random variable is similar to that of usual Geometric distribution if $a=0$ and $d=1$. As we proposed generalization of traditional Geometric distribution, it is the necessary condition that it reduces to traditional distribution if the random variable posses the range 0 to $\infty$.

Definition 2.2.1: A random variable $X$ is said to have a generalized geometric distribution if it has the following probability mass function

$P(x ; a, n, d, p)=q^{x} \frac{\left(1-q^{d}\right)}{q^{a}} \quad ; x=a, a+d, a+2 d, \cdots, a+n d$

where, $a$ is a non-negative integer representing minimum number of failure, $d$ is a positive integer indicating concentration of failure per trail and $n>0$ is an integer representing the total number of trails such that $a=$ 0 if $n=0$ and $p$ is the probability of success such that $p+q=1$ are the parameters of the distribution. The probability function $q^{x} \frac{\left(1-q^{d}\right)}{q^{a}}$ provides the probability of getting the $d$ successes following maximum of $\{a+n d\}$ failures in $n$ trials.

The function in Equation 2.2.1 is a probability function as it satisfies the following properties of probability function for several values of the parameters.

i) $P(x ; a, n, d, p) \geq 0$

ii) $\sum_{x=a}^{a+n d} P(x ; a, n, d, p)=1$

The probability function reduces to $p q^{x}$, where $x=0,1,2, \cdots, \infty$ if $a=0$ and $d=1$. Thus, the name generalized Geometric and traditional one is considered as the special case of the suggested distribution. The other distributional properties are examined below:

Theorem 2.2.1: Prove that the moment generating function of generalized Geometric distribution is

$M_{X}(t)=\frac{\left(1-q^{d}\right) e^{t a}}{\left[1-\left(q e^{t}\right)^{d}\right]}$

The proof of the theorem is presented in the appendix.

Theorem 2.2.2: The first four raw moments of the generalized Geometric distribution are respectively

$\mu_{1}^{\prime}=a+\frac{d q^{d}}{\left(1-q^{d}\right)}$

which is the mean of the distribution.

$\mu_{2}^{\prime}=a^{2}+\frac{a d}{\left(1-q^{d}\right)}+\frac{d(a+d) q^{d}}{\left(1-q^{d}\right)}+\frac{2\left(d q^{d}\right)^{2}}{\left(1-q^{d}\right)^{2}}$ 


$$
\begin{gathered}
\mu_{3}^{\prime}=a^{3}+\frac{2 a^{2} d q^{d}}{\left(1-q^{d}\right)}+\frac{a^{2} d}{\left(1-q^{d}\right)}+\frac{a d^{2}}{\left(1-q^{d}\right)}+\frac{2 a d^{2} q^{d}}{\left(1-q^{d}\right)^{2}}+\frac{2 a d^{2} q^{d}}{\left(1-q^{d}\right)}+\frac{d^{3} q^{d}}{\left(1-q^{d}\right)}+\frac{4 a d^{2} q^{2 d}}{\left(1-q^{d}\right)^{2}}+\frac{6 d^{3} q^{2 d}}{\left(1-q^{d}\right)^{2}} \\
+\frac{6 d^{3} q^{3 d}}{\left(1-q^{d}\right)^{3}} \\
\mu_{4}^{\prime}=a^{4}+\frac{a^{4} d q^{d}}{\left(1-q^{d}\right)}+\frac{a^{3} d q^{d}}{\left(1-q^{d}\right)}+\frac{a^{2} d^{2} q^{d}}{\left(1-q^{d}\right)}+\frac{2 a^{2} d^{2} q^{2 d}}{\left(1-q^{d}\right)^{2}}+\frac{a^{3} d}{\left(1-q^{d}\right)}+\frac{2 a^{2} d^{2}}{\left(1-q^{d}\right)}+\frac{a d^{3}}{\left(1-q^{d}\right)}+\frac{2 a^{2} d^{2} q^{d}}{\left(1-q^{d}\right)^{2}} \\
+\frac{2 a d^{3} q^{d}}{\left(1-q^{d}\right)^{2}}+\frac{a^{3} d q^{d}}{\left(1-q^{d}\right)}+\frac{3 a^{2} d^{2} q^{d}}{\left(1-q^{d}\right)}+\frac{3 a d^{3} q^{d}}{\left(1-q^{d}\right)}+\frac{d^{4} q^{d}}{\left(1-q^{d}\right)}+\frac{2 a^{2} d^{2} q^{2 d}}{\left(1-q^{d}\right)^{2}}+\frac{4 a d^{3} q^{2 d}}{\left(1-q^{d}\right)^{2}} \\
+\frac{2 d^{4} q^{2 d}}{\left(1-q^{d}\right)^{2}}+\frac{2 a^{2} d^{2} q^{d}}{\left(1-q^{d}\right)^{2}}+\frac{4 a d^{3} q^{d}}{\left(1-q^{d}\right)^{2}}+\frac{6 a d^{3} q^{2 d}}{\left(1-q^{d}\right)^{3}}+\frac{4 a^{2} d^{2} q^{2 d}}{\left(1-q^{d}\right)^{2}}+\frac{14 a d^{3} q^{2 d}}{\left(1-q^{d}\right)^{2}}+\frac{12 d^{4} q^{2 d}}{\left(1-q^{d}\right)^{2}} \\
+\frac{12 a d^{3} q^{3 d}}{\left(1-q^{d}\right)^{3}}+\frac{18 d^{4} q^{3 d}}{\left(1-q^{d}\right)^{3}}+\frac{6 a d^{3} q^{3}}{\left(1-q^{d}\right)^{3}}+\frac{18 d^{4} q^{3 d}}{\left(1-q^{d}\right)^{3}}+\frac{24 d^{4} q^{4 d}}{\left(1-q^{d}\right)^{4}}
\end{gathered}
$$

Theorem 2.2.3: The first four central moments of the generalized Geometric distribution are respectively

$$
\begin{aligned}
& \mu_{1}=\mu_{1}^{\prime}=a+\frac{d q^{d}}{\left(1-q^{d}\right)} \\
& \begin{array}{l}
V(X)=\mu_{2}=a d+\frac{d^{2} q^{d}}{\left(1-q^{d}\right)^{2}} \\
\mu_{3}=-2 a^{2} d+\frac{a d^{2}}{\left(1-q^{d}\right)}\left(1-2 q^{d}\right)+\frac{d^{3} q^{d}}{\left(1-q^{d}\right)^{3}}\left(1+q^{d}\right) \\
\mu_{4}=\frac{a^{4} d q^{d}}{\left(1-q^{d}\right)}-\frac{4 d^{2} q^{2 d}}{\left(1-q^{d}\right)^{2}}+\frac{a^{3} d}{\left(1-q^{d}\right)}\left(3-4 q^{d}\right)-\frac{2 a^{2} d^{2}}{\left(1-q^{d}\right)^{2}}\left(1-4 q^{d}+q^{2 d}\right) \\
\quad+\frac{a d^{3}}{\left(1-q^{d}\right)^{2}}\left(1+4 q^{d}-q^{2 d}\right)+\frac{d^{4} q^{d}}{\left(1-q^{d}\right)^{4}}\left(1+7 q^{d}+q^{2 d}\right)
\end{array}
\end{aligned}
$$

The major part of the proof of the Theorem 2.2.2 and Theorem 2.2.3 are given in Appendix.

Theorem 2.2.4: The measures of skewness and kurtosis of generalized geometric distribution.

The Karl Pearson's measures of kurtosis can be calculated based on central moments derived in Theorem 2.2.3.

Theorem 2.2.5 Find the MLE estimator of the parameters of generalized geometric distribution.

Proof: The likelihood function of generalized geometric distribution itself is a probability mass function and which is

$$
\begin{gathered}
L= \\
q^{x} \frac{\left(1-q^{d}\right)}{q^{a}}
\end{gathered}
$$


Taking logarithm in both sides of the equation, we have

$$
\ln L=x \ln q+\ln \left(1-q^{d}\right)-a \ln q
$$

Differentiating equation (2.2.12) with respect to $q$ and equating to zero, we have

$$
\begin{aligned}
& \frac{\delta}{\delta q}(\ln L)=\frac{\delta}{\delta q}\left[x \ln q+\ln \left(1-q^{d}\right)-a \ln q\right]=0 \\
& =>\frac{x}{\hat{q}}-\frac{d \hat{q}^{d-1}}{\left(1-\hat{q}^{d}\right)}-\frac{a}{\hat{q}}=0 \\
& =>\frac{x\left(1-\hat{q}^{d}\right)-d \hat{q}^{d}-a\left(1-\hat{q}^{d}\right)}{\hat{q}\left(1-\hat{q}^{d}\right)}=0 \\
& =>x-x \hat{q}^{d}-d \hat{q}^{d}-a+a \hat{q}^{d}=0 \\
& =>\hat{q}^{d}(a-d-x)=a-x \\
& =>\hat{q}^{d}=\frac{a-x}{a-d-x} \\
& =>\hat{q}=\left(\frac{a-x}{a-d-x}\right)^{\frac{1}{d}}
\end{aligned}
$$

Which is the MLE estimator of probability of failure before first success.

Hence, the MLE estimator of probability of success after $x$ failure will be

$$
\hat{p}=1-\hat{q}=1-\left(\frac{a-x}{a-d-x}\right)^{\frac{1}{d}}
$$

\section{Discussion and Conclusion}

In probability theory and statistics Poisson distribution and Geometric distribution have great importance. In both the distribution, the value of the random variable ranges from 0 to $\infty$ with a constant increment of 1 for each trail. We proposed the generalized version of both the distribution where the number of occurrences can be expressed by an arithmetic progression $a+n d$. It is shown that the traditional forms of the distributions are the special case of the proposed distributions. Thus, both the generalized Poisson and generalized Geometric distribution can be applied in the cases where their traditional distribution is the only way. In parallel, the proposed distributions facilitates to solving the probability of the certain value of the random variable having infinitely many sequences other than traditional sequence. In this context, the scope of the proposed distribution is much wider than their traditional form. In addition, some of the distributional properties are derived and examined for both of the suggested distributions. Overall, the generalized Poisson and generalized Geometric distributions may play a critical and vital role in the distribution theory and thus in the complicated real life problems.

\section{Reference}

[1] P. C. Consul and G. C Jain, A Generalization of the Poisson Distribution, Technometrics. 15(4) 1973 791-799.

[2] P. C. Consul, Generalized Poisson Distribution: Properties and Applications, (Statistics. Text Book and Monograms, New York: Marcel Dekker Inc. R. Loren and D. B. Benson (eds.), 1989).

[3] B. Lerner, A. Lone, and M. Rao, On generalized Poisson distributions, Probab. Math. Statist. 17(2) $1997377-385$.

[4] D. L. Nelson, Some Remarks on Generalizations of the Negative Binomial and Poisson Distributions, Technometrics. 17(1) 1975 135-136. 
[5] A. E. Paul, A Generalized Compound Poisson Model for Consumer Purchase Panel Data Analysis, J. Amer. Statist. Assoc. 73(364) 1978 706-713.

[6] X. S. Lin, Generalized Poisson Models and Their Applications in Insurance and Finance, J. Amer. Statist. Assoc. 99(466) 2004 563.

[7] P. Hagmark, An Exceptional Generalization of the Poisson Distribution. Open J. Statist. 2 2012 313-318.

[8] A. Mishra, A Generalization of Geometric Series Distribution, J. BiharMath. Soc. 6 1982 14-17.

[9] G. C. Jain and P. C. Consul, A generalized negative binomial distribution, SIAM J. Appl. Math. 21(4) 1971 501-513.

[10] A. N. Philippou, C. Georghiou and G. N. Philippou, A Generalized Geometric Distribution and some of its Properties. Statist. Probab. Lett. 1(4) 1983 171-175.

[11] A. N. Philippou and A. A. Muwafi, Waiting for the kth consecutive success and the Fibonacci sequence of order k, The Fibonacci Quart. 20 (1) 1982 28-32.

[12] R. A. Kempton, A generalized form of Fisher's logarithmic series, Biometrika. 62(1) 1975 28-38.

[13] R. C. Tripathi and R. C. Gupta, A generalization of the log-series distribution, Comm. Statist. Theory Methods 14(8) 1985 17791799.

[14] R. C. Tripathi, R. C. Gupta and T. G. White, Some Generalizations of the Geometric Distribution. Sankhya B 49(3) 1987218 223

[15] E. Gomez-Deniz, Another generalization of the geometric distribution. Test 19(2) 2010 399-415.

[16] A. N. Marshall, and I. Olkin, A new method for adding a parameter to a family of distributions with applications to the exponential and Weibull families, Biometrika. 84(3) 1997 641-652.

[17] M. Nassar and M. Nada, A new generalization of the Pareto-geometric distribution. J. Egyptian Math. Soc. 21(2) 2013 148-155.

\section{Appendix}

Derivation of Generalized Poisson Distribution: Under the assumptions made above, the mass function of the generalized Binomial distribution can be written as

$$
\begin{aligned}
P(x ; a, n, d, p) & =\frac{\left(\begin{array}{c}
a+n d \\
x
\end{array}\right) p^{x} q^{a+n d-x}}{\sum_{x=a}^{a+n d}\left(\begin{array}{c}
a+n d \\
x
\end{array}\right) p^{x} q^{a+n d-x}} ; x=a, a+d, a+2 d, \cdots, a+n d \\
& =\frac{\frac{(a+n d) !}{x !(a+n d-x) !}\left\{\frac{\lambda}{(a+n d)}\right\}^{x}\left\{1-\frac{\lambda}{(a+n d)}\right\}^{a+n d-x}}{\sum_{x=a}^{a+n d} \frac{(a+n d) !}{x !(a+n d-x) !}\left\{\frac{\lambda}{(a+n d)}\right\}^{x}\left\{1-\frac{\lambda}{(a+n d)}\right\}^{a+n d-x}} \\
& =\frac{\frac{\lambda^{x}}{x !}\left\{1-\frac{\lambda}{(a+n d)}\right\}^{-x}\left\{1-\frac{\lambda}{(a+n d)}\right\}^{a+n d} \frac{(a+n d) !}{(a+n d)^{x}(a+n d-x) !}}{\sum_{x=a}^{a+n d} \frac{\lambda^{x}}{x !}\left\{1-\frac{\lambda}{(a+n d)}\right\}^{-x}\left\{1-\frac{\lambda}{(a+n d)}\right\}^{a+n d} \frac{(a+n d) !}{(a+n d)^{x}(a+n d-x) !}}
\end{aligned}
$$

Here it is noted that if $n \rightarrow \infty$ then definitely $a+n d \rightarrow \infty$.

Now, for fixed $x \lim _{n \rightarrow \infty}\left\{1-\frac{\lambda}{(a+n d)}\right\}^{-x}=1$ and

$$
\begin{aligned}
& \lim _{n \rightarrow \infty} \frac{(a+n d) !}{(a+n d)^{x}(a+n d-x) !} \\
& =\lim _{n \rightarrow \infty} \frac{(a+n d)(a+n d-1)(a+n d-2) \cdots(a+n d-(x-1))(a+n d-x) !}{(a+n d)^{x}(a+n d-x) !} \\
& =\lim _{n \rightarrow \infty} \frac{(a+n d)(a+n d-1)(a+n d-2) \cdots(a+n d-(x-1))}{(a+n d)^{x}}
\end{aligned}
$$




$$
\begin{aligned}
& =\lim _{n \rightarrow \infty} \frac{(a+n d)^{x}\left(1-\frac{1}{a+n d}\right)\left(1-\frac{2}{a+n d}\right) \cdots\left(1-\frac{x-1}{a+n d}\right)}{(a+n d)^{x}} \\
& =\lim _{n \rightarrow \infty}\left(1-\frac{1}{a+n d}\right)\left(1-\frac{2}{a+n d}\right) \cdots\left(1-\frac{x-1}{a+n d}\right) \\
& =1
\end{aligned}
$$

Again, $\lim _{n \rightarrow \infty}\left\{1-\frac{\lambda}{(a+n d)}\right\}^{a+n d}=e^{\frac{-\lambda}{(a+n d)}(a+n d)}=e^{-\lambda}$

Hence,

$\lim _{n \rightarrow \infty} P(x ; a, n, d, p)=P(x ; \lambda, a, n, d)=\frac{\frac{\lambda^{x}}{x !} e^{-\lambda}}{\sum_{x=a}^{a+n d} \frac{\lambda^{x}}{x !} e^{-\lambda}}=\frac{\frac{\lambda^{x}}{x !}}{\sum_{x=a}^{a+n d} \frac{\lambda^{x}}{x !}}=\frac{\frac{\lambda^{x}}{x !}}{\sum_{n=0}^{\infty} \frac{\lambda^{a+n d}}{(a+n d) !}}$

which is the probability mass function of Generalized Poisson distribution with parameters $\lambda \geq 0, a \geq 0, d>$ 0 and $n \geq 0$.

Proof of Theorem 2.2.1: According to the definition of moment generating function

$$
\begin{aligned}
& M_{X}(t)=E\left(e^{t X}\right)=\sum_{x=a}^{a+n d} e^{t X} P(x ; a, n, d, p) \\
& =>M_{X}(t)=\sum_{x=a}^{a+n d} e^{t X} q^{x} \frac{\left(1-q^{d}\right)}{q^{a}} \\
& =>M_{X}(t)=\sum_{x=a}^{a+n d}\left(q e^{t}\right)^{x} \frac{\left(1-q^{d}\right)}{q^{a}} \\
& =>M_{X}(t)=\frac{\left(1-q^{d}\right)}{q^{a}} \sum_{x=a}^{a+n d}\left(q e^{t}\right)^{x} \\
& =>M_{X}(t)=\frac{\left(1-q^{d}\right)}{q^{a}}\left[\left(q e^{t}\right)^{a}+\left(q e^{t}\right)^{a+d}+\left(q e^{t}\right)^{a+2 d}+\left(q e^{t}\right)^{a+3 d}+\cdots\right] \\
& =>M_{X}(t)=\frac{\left(1-q^{d}\right)}{q^{a}}\left(q e^{t}\right)^{a}\left[1+\left(q e^{t}\right)^{d}+\left(q e^{t}\right)^{2 d}+\left(q e^{t}\right)^{3 d}+\cdots\right] \\
& =>M_{X}(t)=\left(1-q^{d}\right) e^{t a}\left[1+\left(q e^{t}\right)^{d}+\left[\left(q e^{t}\right)^{d}\right]^{2}+\left[\left(q e^{t}\right)^{d}\right]^{3}+\cdots\right] \\
& =>M_{X}(t)=\left(1-q^{d}\right) e^{t a}\left[1-\left(q e^{t}\right)^{d}\right]^{-1} \\
& \therefore M_{X}(t)=\frac{\left(1-q^{d}\right) e^{t a}}{\left[1-\left(q e^{t}\right)^{d}\right]}
\end{aligned}
$$

Which is the moment generating function of generalized Geometric distribution.

\section{Proof of Theorem 2.2.2:}

$\underline{1^{\text {st }} \text { raw moment: }}$ 
The $1^{\text {st }}$ raw moment or mean of the distribution can be calculated from its moment generating function (see Equation 2.2.2) by differentiating it with respect to $t$ and equating $t=0$. That is

$$
\begin{aligned}
& E(X)=\mu_{1}^{\prime}=\left.\frac{d}{d t}\left[M_{X}(t)\right]\right|_{t=0} \\
& =\left.\frac{d}{d t}\left[\frac{\left(1-q^{d}\right) e^{t a}}{\left[1-\left(q e^{t}\right)^{d}\right]}\right]\right|_{t=0} \\
& =\left.\left[\left(1-q^{d}\right) \frac{\left[1-\left(q e^{t}\right)^{d}\right] a e^{t a}+d e^{t a}\left(q e^{t}\right)^{d}}{\left[1-\left(q e^{t}\right)^{d}\right]^{2}}\right]\right|_{t=0} \\
& =\left.\left[\left(1-q^{d}\right)\left\{\frac{a e^{t a}}{\left[1-\left(q e^{t}\right)^{d}\right]}+\frac{d q^{d} e^{(a+d) t}}{\left[1-\left(q e^{t}\right)^{d}\right]^{2}}\right\}\right]\right|_{t=0} \\
& =\left(1-q^{d}\right)\left\{\frac{a}{\left(1-q^{d}\right)}+\frac{d q^{d}}{\left(1-q^{d}\right)^{2}}\right\} \\
& =a+\frac{d q^{d}}{\left(1-q^{d}\right)}
\end{aligned}
$$

$2^{\text {nd }}$ raw moment:

$$
\begin{aligned}
\mu_{2}^{\prime} & =\left.\frac{d^{2}}{d t^{2}}\left[M_{X}(t)\right]\right|_{t=0} \\
& =\left.\left(1-q^{d}\right) \frac{d}{d t}\left\{\frac{a e^{t a}}{\left[1-\left(q e^{t}\right)^{d}\right]}+\frac{d q^{d} e^{(a+d) t}}{\left[1-\left(q e^{t}\right)^{d}\right]^{2}}\right\}\right|_{t=0} \\
& =\left(1-q^{d}\right)\left\{\frac{\left[1-\left(q e^{t}\right)^{d}\right] a^{2} e^{t a}-a e^{t a}\left(-d\left(q e^{t}\right)^{d}\right)}{\left[1-\left(q e^{t}\right)^{d}\right]^{2}}\right. \\
& \left.+\frac{\left[1-\left(q e^{t}\right)^{d}\right]^{2} d q^{d}(a+d) e^{(a+d) t}-d q^{d} e^{(a+d) t} 2\left[1-\left(q e^{t}\right)^{d}\right]\left(-d\left(q e^{t}\right)^{d}\right)}{\left[1-\left(q e^{t}\right)^{d}\right]^{4}}\right\}\left.\right|_{t=0} \\
& =a^{2}+\frac{a d}{\left(1-q^{d}\right)}+\frac{d(a+d) q^{d}}{\left(1-q^{d}\right)}+\frac{2\left(d q^{d}\right)^{2}}{\left(1-q^{d}\right)^{2}}
\end{aligned}
$$

$\underline{3^{\text {rd }} \text { raw moment: }}$

$$
\begin{aligned}
& \mu_{3}^{\prime}=\left.\frac{d^{3}}{d t^{3}}\left[M_{X}(t)\right]\right|_{t=0} \\
& =\left.\left(1-q^{d}\right) \frac{d}{d t}\left\{\frac{a^{2} e^{t a}}{\left[1-\left(q e^{t}\right)^{d}\right]}+\frac{a d e^{(a+d) t}}{\left[1-\left(q e^{t}\right)^{d}\right]^{2}}+\frac{d(a+d) q^{d} e^{(a+d) t}}{\left[1-\left(q e^{t}\right)^{d}\right]^{2}}+\frac{2\left(d q^{d}\right)^{2} e^{(a+2 d) t}}{\left[1-\left(q e^{t}\right)^{d}\right]^{3}}\right\}\right|_{t=0} \\
& =\left.\left(1-q^{d}\right) \frac{d}{d t}\left\{\frac{a^{2} e^{t a}}{\left[1-\left(q e^{t}\right)^{d}\right]}+\left[a d+d(a+d) q^{d}\right] \frac{e^{(a+d) t}}{\left[1-\left(q e^{t}\right)^{d}\right]^{2}}+\frac{2\left(d q^{d}\right)^{2} e^{(a+2 d) t}}{\left[1-\left(q e^{t}\right)^{d}\right]^{3}}\right\}\right|_{t=0}
\end{aligned}
$$




$$
\begin{aligned}
& =\left(1-q^{d}\right)\left\{\frac{\left[1-\left(q e^{t}\right)^{d}\right] a^{3} e^{t a}-a^{2} e^{t a}\left(-d\left(q e^{t}\right)^{d}\right)}{\left[1-\left(q e^{t}\right)^{d}\right]^{2}}\right. \\
& +\left[a d+d(a+d) q^{d}\right] \frac{\left[1-\left(q e^{t}\right)^{d}\right]^{2}(a+d) e^{(a+d) t}-e^{(a+d) t}\left[2\left[1-\left(q e^{t}\right)^{d}\right]\left(-d\left(q e^{t}\right)^{d}\right)\right]}{\left[1-\left(q e^{t}\right)^{d}\right]^{4}} \\
& \left.+\frac{\left[1-\left(q e^{t}\right)^{d}\right]^{3} 2\left(d q^{d}\right)^{2}(a+2 d) e^{(a+2 d) t}-2\left(d q^{d}\right)^{2} e^{(a+2 d) t}\left[3\left[1-\left(q e^{t}\right)^{d}\right]^{2}\left(-d\left(q e^{t}\right)^{d}\right)\right]}{\left[1-\left(q e^{t}\right)^{d}\right]^{6}}\right\}\left.\right|_{t=0} \\
& =\left(1-q^{d}\right)\left\{\frac{a^{3}}{\left(1-q^{d}\right)}+\frac{a^{2} d q^{d}}{\left(1-q^{d}\right)^{2}}+\left[a d+d(a+d) q^{d}\right]\left[\frac{(a+d)}{\left(1-q^{d}\right)^{2}}+\frac{2 d q^{d}}{\left(1-q^{d}\right)^{3}}\right]+\frac{2\left(d q^{d}\right)^{2}(a+2 d)}{\left(1-q^{d}\right)^{3}}\right. \\
& \left.+\frac{6\left(d q^{d}\right)^{3}}{\left(1-q^{d}\right)^{4}}\right\} \\
& =a^{3}+\frac{a^{2} d q^{d}}{\left(1-q^{d}\right)}+\frac{a d(a+d)}{\left(1-q^{d}\right)}+\frac{2 a d^{2} q^{d}}{\left(1-q^{d}\right)^{2}}+\frac{d(a+d)^{2} q^{d}}{\left(1-q^{d}\right)}+\frac{2(a+d)\left(d q^{d}\right)^{2}}{\left(1-q^{d}\right)^{2}}+\frac{2(a+2 d)\left(d q^{d}\right)^{2}}{\left(1-q^{d}\right)^{2}} \\
& +\frac{6\left(d q^{d}\right)^{3}}{\left(1-q^{d}\right)^{3}} \\
& =a^{3}+\frac{2 a^{2} d q^{d}}{\left(1-q^{d}\right)}+\frac{a^{2} d}{\left(1-q^{d}\right)}+\frac{a d^{2}}{\left(1-q^{d}\right)}+\frac{2 a d^{2} q^{d}}{\left(1-q^{d}\right)^{2}}+\frac{2 a d^{2} q^{d}}{\left(1-q^{d}\right)}+\frac{d^{3} q^{d}}{\left(1-q^{d}\right)}+\frac{4 a d^{2} q^{2 d}}{\left(1-q^{d}\right)^{2}}+\frac{6 d^{3} q^{2 d}}{\left(1-q^{d}\right)^{2}} \\
& +\frac{6 d^{3} q^{3 d}}{\left(1-q^{d}\right)^{3}} \\
& \mu_{4}^{\prime}=\left.\frac{d^{4}}{d t^{4}}\left[M_{X}(t)\right]\right|_{t=0} \\
& =\left(1-q^{d}\right) \frac{d}{d t}\left\{\frac{a^{3} e^{t a}}{\left[1-\left(q e^{t}\right)^{d}\right]}+\frac{a^{2} d q^{d} e^{(a+d) t}}{\left[1-\left(q e^{t}\right)^{d}\right]^{2}}+\left[a d+d(a+d) q^{d}\right]\left[\frac{(a+d) e^{(a+d) t}}{\left[1-\left(q e^{t}\right)^{d}\right]^{2}}+\frac{2 d q^{d} e^{(a+2 d) t}}{\left[1-\left(q e^{t}\right)^{d}\right]^{3}}\right]\right. \\
& \left.+\frac{2\left(d q^{d}\right)^{2}(a+2 d) e^{(a+2 d) t}}{\left[1-\left(q e^{t}\right)^{d}\right]^{3}}+\frac{6\left(d q^{d}\right)^{3} e^{(a+3 d) t}}{\left[1-\left(q e^{t}\right)^{d}\right]^{4}}\right\}\left.\right|_{t=0} \\
& =\left(1-q^{d}\right) \frac{d}{d t}\left\{\frac{a^{3} e^{t a}}{\left[1-\left(q e^{t}\right)^{d}\right]}+\left[a^{2} d q^{d}+a d(a+d)+d(a+d)^{2} q^{d}\right] \frac{e^{(a+d) t}}{\left[1-\left(q e^{t}\right)^{d}\right]^{2}}\right. \\
& +\left[2 a d^{2} q^{d}+2(a+d)\left(d q^{d}\right)^{2}+2\left(d q^{d}\right)^{2}(a+2 d)\right] \frac{e^{(a+2 d) t}}{\left[1-\left(q e^{t}\right)^{d}\right]^{3}} \\
& \left.+\frac{6\left(d q^{d}\right)^{3} e^{(a+3 d) t}}{\left[1-\left(q e^{t}\right)^{d}\right]^{4}}\right\}\left.\right|_{t=0}
\end{aligned}
$$




$$
\begin{aligned}
& =\left(1-q^{d}\right)\left\{\frac{\left[1-\left(q e^{t}\right)^{d}\right] a^{4} e^{t a}-a^{4} e^{t a}\left(-d\left(q e^{t}\right)^{d}\right)}{\left[1-\left(q e^{t}\right)^{d}\right]^{2}}\right. \\
& +\left[a^{2} d q^{d}+a d(a+d)+d(a+d)^{2} q^{d}\right] \frac{\left[1-\left(q e^{t}\right)^{d}\right]^{2}(a+d) e^{(a+d) t}-e^{(a+d) t} 2\left[1-\left(q e^{t}\right)^{d}\right]\left(-d\left(q e^{t}\right)^{d}\right)}{\left[1-\left(q e^{t}\right)^{d}\right]^{4}} \\
& +\left[2 a d^{2} q^{d}+2(2 a+3 d)\left(d q^{d}\right)^{2}\right] \frac{\left[1-\left(q e^{t}\right)^{d}\right]^{3}(a+2 d) e^{(a+2 d) t}-e^{(a+2 d) t} 3\left[1-\left(q e^{t}\right)^{d}\right]^{2}\left(-d\left(q e^{t}\right)^{d}\right)}{\left[1-\left(q e^{t}\right)^{d}\right]^{6}} \\
& \left.+\frac{\left[1-\left(q e^{t}\right)^{d}\right]^{4} 6\left(d q^{d}\right)^{3}(a+3 d) e^{(a+3 d) t}-6\left(d q^{d}\right)^{3} e^{(a+3 d) t} 4\left[1-\left(q e^{t}\right)^{d}\right]^{3}\left(-d\left(q e^{t}\right)^{d}\right)}{\left[1-\left(q e^{t}\right)^{d}\right]^{8}}\right\}\left.\right|_{t=0} \\
& =a^{4}+\frac{a^{4} d q^{d}}{\left(1-q^{d}\right)}+\frac{a^{3} d q^{d}}{\left(1-q^{d}\right)}+\frac{a^{2} d^{2} q^{d}}{\left(1-q^{d}\right)}+\frac{2 a^{2} d^{2} q^{2 d}}{\left(1-q^{d}\right)^{2}}+\frac{a^{3} d}{\left(1-q^{d}\right)}+\frac{2 a^{2} d^{2}}{\left(1-q^{d}\right)}+\frac{a d^{3}}{\left(1-q^{d}\right)}+\frac{2 a^{2} d^{2} q^{d}}{\left(1-q^{d}\right)^{2}}+\frac{2 a d^{3} q^{d}}{\left(1-q^{d}\right)^{2}}+\frac{a^{3} d q^{d}}{\left(1-q^{d}\right)} \\
& +\frac{3 a^{2} d^{2} q^{d}}{\left(1-q^{d}\right)}+\frac{3 a d^{3} q^{d}}{\left(1-q^{d}\right)}+\frac{d^{4} q^{d}}{\left(1-q^{d}\right)}+\frac{2 a^{2} d^{2} q^{2 d}}{\left(1-q^{d}\right)^{2}}+\frac{4 a d^{3} q^{2 d}}{\left(1-q^{d}\right)^{2}}+\frac{2 d^{4} q^{2 d}}{\left(1-q^{d}\right)^{2}}+\frac{2 a^{2} d^{2} q^{d}}{\left(1-q^{d}\right)^{2}}+\frac{4 a d^{3} q^{d}}{\left(1-q^{d}\right)^{2}}+\frac{6 a d^{3} q^{2 d}}{\left(1-q^{d}\right)^{3}}+\frac{4 a^{2} d^{2} q^{2 d}}{\left(1-q^{d}\right)^{2}} \\
& +\frac{14 a d^{3} q^{2 d}}{\left(1-q^{d}\right)^{2}}+\frac{12 d^{4} q^{2 d}}{\left(1-q^{d}\right)^{2}}+\frac{12 a d^{3} q^{3 d}}{\left(1-q^{d}\right)^{3}}+\frac{18 d^{4} q^{3 d}}{\left(1-q^{d}\right)^{3}}+\frac{6 a d^{3} q^{3 d}}{\left(1-q^{d}\right)^{3}}+\frac{18 d^{4} q^{3 d}}{\left(1-q^{d}\right)^{3}} \\
& +\frac{24 d^{4} q^{4 d}}{\left(1-q^{d}\right)^{4}}
\end{aligned}
$$

\section{Proof of Theorem 2.2.3:}

The central moments can be easily derived from the relationship between the raw and central moments once we have raw moments. Thus, we do provide here shortly the derivation of $3^{\text {rd }}$ and $4^{\text {th }}$ central moments only.

\section{$\underline{3^{\text {rd }} \text { Central Moment }}$}

$$
\begin{aligned}
& \mu_{3}=\mu_{3}^{\prime}-3 \mu_{2}^{\prime} \mu_{1}^{\prime}+2 \mu_{1}^{\prime 3} \\
& =a^{3}+\frac{2 a^{2} d q^{d}}{\left(1-q^{d}\right)}+\frac{a^{2} d}{\left(1-q^{d}\right)}+\frac{a d^{2}}{\left(1-q^{d}\right)}+\frac{2 a d^{2} q^{d}}{\left(1-q^{d}\right)^{2}}+\frac{2 a d^{2} q^{d}}{\left(1-q^{d}\right)}+\frac{d^{3} q^{d}}{\left(1-q^{d}\right)}+\frac{4 a d^{2} q^{2 d}}{\left(1-q^{d}\right)^{2}}+\frac{6 d^{3} q^{2 d}}{\left(1-q^{d}\right)^{2}}+\frac{6 d^{3} q^{3 d}}{\left(1-q^{d}\right)^{3}} \\
& -3 a^{3}-\frac{3 a^{2} d q^{d}}{\left(1-q^{d}\right)}-\frac{3 a^{2} d}{\left(1-q^{d}\right)}-\frac{3 a d^{2} q^{d}}{\left(1-q^{d}\right)^{2}}-\frac{3 a^{2} d q^{d}}{\left(1-q^{d}\right)}-\frac{3 a d^{2} q^{d}}{\left(1-q^{d}\right)}-\frac{3 a d^{2} q^{2 d}}{\left(1-q^{d}\right)^{2}}-\frac{3 d^{3} q^{2 d}}{\left(1-q^{d}\right)^{2}} \\
& -\frac{6 a d^{2} q^{2 d}}{\left(1-q^{d}\right)^{2}}-\frac{6 d^{3} q^{3 d}}{\left(1-q^{d}\right)^{3}}+2 a^{3}+\frac{6 a^{2} d q^{d}}{\left(1-q^{d}\right)}+\frac{6 a d^{2} q^{2 d}}{\left(1-q^{d}\right)^{2}}+\frac{2 d^{3} q^{3 d}}{\left(1-q^{d}\right)^{3}} \\
& =\frac{2 a^{2} d q^{d}}{\left(1-q^{d}\right)}-\frac{2 a^{2} d}{\left(1-q^{d}\right)}+\frac{a d^{2}}{\left(1-q^{d}\right)}-\frac{a d^{2} q^{d}}{\left(1-q^{d}\right)^{2}}-\frac{a d^{2} q^{d}}{\left(1-q^{d}\right)}+\frac{d^{3} q^{d}}{\left(1-q^{d}\right)}+\frac{a d^{2} q^{2 d}}{\left(1-q^{d}\right)^{2}}+\frac{3 d^{3} q^{2 d}}{\left(1-q^{d}\right)^{2}} \\
& +\frac{2 d^{3} q^{3 d}}{\left(1-q^{d}\right)^{3}} \\
& =\frac{2 a^{2} d}{\left(1-q^{d}\right)}\left(q^{d}-1\right)+\frac{a d^{2}}{\left(1-q^{d}\right)^{2}}\left\{1-q^{d}-q^{d}-q^{d}\left(1-q^{d}\right)+q^{2 d}\right\} \\
& +\frac{d^{3} q^{d}}{\left(1-q^{d}\right)^{3}}\left\{\left(1-q^{d}\right)^{2}+3 q^{d}\left(1-q^{d}\right)+2 q^{2 d}\right\} \\
& =-2 a^{2} d+\frac{a d^{2}}{\left(1-q^{d}\right)^{2}}\left\{1-3 q^{d}+2 q^{2 d}\right\}+\frac{d^{3} q^{d}}{\left(1-q^{d}\right)^{3}}\left\{1-2 q^{d}+q^{2 d}+3 q^{d}-3 q^{2 d}+2 q^{2 d}\right\} \\
& =-2 a^{2} d+\frac{a d^{2}}{\left(1-q^{d}\right)^{2}}\left\{1-q^{d}-2 q^{d}+2 q^{2 d}\right\}+\frac{d^{3} q^{d}}{\left(1-q^{d}\right)^{3}}\left\{1+q^{d}\right\}
\end{aligned}
$$




$$
\begin{aligned}
& =-2 a^{2} d+\frac{a d^{2}}{\left(1-q^{d}\right)^{2}}\left(1-q^{d}\right)\left(1-2 q^{d}\right)+\frac{d^{3} q^{d}}{\left(1-q^{d}\right)^{3}}\left(1+q^{d}\right) \\
& =-2 a^{2} d+\frac{a d^{2}}{\left(1-q^{d}\right)}\left(1-2 q^{d}\right)+\frac{d^{3} q^{d}}{\left(1-q^{d}\right)^{3}}\left(1+q^{d}\right)
\end{aligned}
$$

\section{$4^{\text {th }}$ Central Moment}

$$
\begin{aligned}
& \mu_{4}=\mu_{4}^{\prime}-4 \mu_{3}^{\prime} \mu_{1}^{\prime}+6 \mu_{2}^{\prime} \mu_{1}^{\prime 2}-3 \mu_{1}^{\prime 4} \\
& =>\mu_{4}=a^{4}+\frac{a^{4} d q^{d}}{\left(1-q^{d}\right)}+\frac{a^{3} d q^{d}}{\left(1-q^{d}\right)}+\frac{a^{2} d^{2} q^{d}}{\left(1-q^{d}\right)}+\frac{2 a^{2} d^{2} q^{2 d}}{\left(1-q^{d}\right)^{2}}+\frac{a^{3} d}{\left(1-q^{d}\right)}+\frac{2 a^{2} d^{2}}{\left(1-q^{d}\right)}+\frac{a d^{3}}{\left(1-q^{d}\right)}+\frac{2 a^{2} d^{2} q^{d}}{\left(1-q^{d}\right)^{2}}+\frac{2 a d^{3} q^{d}}{\left(1-q^{d}\right)^{2}} \\
& +\frac{a^{3} d q^{d}}{\left(1-q^{d}\right)}+\frac{3 a^{2} d^{2} q^{d}}{\left(1-q^{d}\right)}+\frac{3 a d^{3} q^{d}}{\left(1-q^{d}\right)}+\frac{d^{4} q^{d}}{\left(1-q^{d}\right)}+\frac{2 a^{2} d^{2} q^{2 d}}{\left(1-q^{d}\right)^{2}}+\frac{4 a d^{3} q^{2 d}}{\left(1-q^{d}\right)^{2}}+\frac{2 d^{4} q^{2 d}}{\left(1-q^{d}\right)^{2}}+\frac{2 a^{2} d^{2} q^{d}}{\left(1-q^{d}\right)^{2}}+\frac{4 a d^{3} q^{d}}{\left(1-q^{d}\right)^{2}} \\
& +\frac{6 a d^{3} q^{2 d}}{\left(1-q^{d}\right)^{3}}+\frac{4 a^{2} d^{2} q^{2 d}}{\left(1-q^{d}\right)^{2}}+\frac{14 a d^{3} q^{2 d}}{\left(1-q^{d}\right)^{2}}+\frac{12 d^{4} q^{2 d}}{\left(1-q^{d}\right)^{2}}+\frac{12 a d^{3} q^{3 d}}{\left(1-q^{d}\right)^{3}}+\frac{18 d^{4} q^{3 d}}{\left(1-q^{d}\right)^{3}}+\frac{6 a d^{3} q^{3 d}}{\left(1-q^{d}\right)^{3}}+\frac{18 d^{4} q^{3 d}}{\left(1-q^{d}\right)^{3}} \\
& +\frac{24 d^{4} q^{4 d}}{\left(1-q^{d}\right)^{4}}-4 a^{4}-\frac{8 a^{3} d q^{d}}{\left(1-q^{d}\right)}-\frac{4 a^{3} d}{\left(1-q^{d}\right)}-\frac{4 a^{2} d^{2}}{\left(1-q^{d}\right)}-\frac{8 a^{2} d^{2} q^{d}}{\left(1-q^{d}\right)^{2}}-\frac{8 a^{2} d^{2} q^{d}}{\left(1-q^{d}\right)}-\frac{4 a d^{3} q^{d}}{\left(1-q^{d}\right)}-\frac{16 a^{2} d^{2} q^{2 d}}{\left(1-q^{d}\right)^{2}} \\
& -\frac{24 a d^{3} q^{2 d}}{\left(1-q^{d}\right)^{2}}-\frac{24 a d^{3} q^{3 d}}{\left(1-q^{d}\right)^{3}}-\frac{4 a^{3} d q^{d}}{\left(1-q^{d}\right)}-\frac{8 a^{2} d^{2} q^{2 d}}{\left(1-q^{d}\right)^{2}}-\frac{4 a^{2} d^{2} q^{d}}{\left(1-q^{d}\right)^{2}}-\frac{4 a d^{3} q^{d}}{\left(1-q^{d}\right)^{2}}-\frac{8 a d^{3} q^{2 d}}{\left(1-q^{d}\right)^{3}}-\frac{8 a d^{3} q^{2 d}}{\left(1-q^{d}\right)^{2}} \\
& -\frac{4 d^{4} q^{2 d}}{\left(1-q^{d}\right)^{2}}-\frac{16 a d^{3} q^{3 d}}{\left(1-q^{d}\right)^{3}}-\frac{24 d^{4} q^{3 d}}{\left(1-q^{d}\right)^{3}}-\frac{24 d^{4} q^{4 d}}{\left(1-q^{d}\right)^{4}}+6 a^{4}+\frac{6 a^{3} d}{\left(1-q^{d}\right)}+\frac{6 a^{3} d q^{d}}{\left(1-q^{d}\right)}+\frac{6 a^{2} d^{2} q^{d}}{\left(1-q^{d}\right)}+\frac{12 a^{2} d^{2} q^{2 d}}{\left(1-q^{d}\right)^{2}} \\
& +\frac{12 a^{3} d q^{d}}{\left(1-q^{d}\right)}+\frac{12 a^{2} d^{2} q^{d}}{\left(1-q^{d}\right)^{2}}+\frac{12 a^{2} d^{2} q^{2 d}}{\left(1-q^{d}\right)^{2}}+\frac{12 a d^{3} q^{2 d}}{\left(1-q^{d}\right)^{2}}+\frac{24 a d^{3} q^{3 d}}{\left(1-q^{d}\right)^{3}}+\frac{6 a^{2} d^{2} q^{2 d}}{\left(1-q^{d}\right)^{2}}+\frac{6 a d^{3} q^{2 d}}{\left(1-q^{d}\right)^{3}}+\frac{6 a d^{3} q^{3 d}}{\left(1-q^{d}\right)^{3}} \\
& +\frac{6 d^{4} q^{3 d}}{\left(1-q^{d}\right)^{3}}+\frac{12 d^{4} q^{4 d}}{\left(1-q^{d}\right)^{4}}-3 a^{4}-\frac{12 a^{3} d q^{d}}{\left(1-q^{d}\right)}-\frac{18 a^{2} d^{2} q^{2 d}}{\left(1-q^{d}\right)^{2}}-\frac{12 a d^{3} q^{3 d}}{\left(1-q^{d}\right)^{3}}-\frac{3 d^{4} q^{4 d}}{\left(1-q^{d}\right)^{4}} \\
& =>\mu_{4}=\frac{a^{4} d q^{d}}{\left(1-q^{d}\right)}-\frac{4 a^{3} d q^{d}}{\left(1-q^{d}\right)}+\frac{2 a^{2} d^{2} q^{d}}{\left(1-q^{d}\right)}-\frac{4 d^{2} q^{2 d}}{\left(1-q^{d}\right)^{2}}+\frac{3 a^{3} d}{\left(1-q^{d}\right)}-\frac{2 a^{2} d^{2}}{\left(1-q^{d}\right)}+\frac{a d^{3}}{\left(1-q^{d}\right)}+\frac{4 a^{2} d^{2} q^{d}}{\left(1-q^{d}\right)^{2}}+\frac{2 a d^{3} q^{d}}{\left(1-q^{d}\right)^{2}}-\frac{a d^{3} q^{d}}{\left(1-q^{d}\right)} \\
& +\frac{d^{4} q^{d}}{\left(1-q^{d}\right)}-\frac{2 a d^{3} q^{2 d}}{\left(1-q^{d}\right)^{2}}+\frac{10 d^{4} q^{2 d}}{\left(1-q^{d}\right)^{2}}+\frac{4 a d^{3} q^{2 d}}{\left(1-q^{d}\right)^{3}}-\frac{4 a d^{3} q^{3 d}}{\left(1-q^{d}\right)^{3}}+\frac{18 d^{4} q^{3 d}}{\left(1-q^{d}\right)^{3}}+\frac{9 d^{4} q^{4 d}}{\left(1-q^{d}\right)^{4}} \\
& =>\mu_{4}=\frac{a^{4} d q^{d}}{\left(1-q^{d}\right)}-\frac{4 a^{3} d q^{d}}{\left(1-q^{d}\right)}-\frac{4 d^{2} q^{2 d}}{\left(1-q^{d}\right)^{2}}+\frac{3 a^{3} d}{\left(1-q^{d}\right)}-\frac{2 a^{2} d^{2}}{\left(1-q^{d}\right)}\left(1-q^{d}\right)+\frac{a d^{3}}{\left(1-q^{d}\right)}\left(1-q^{d}\right)+\frac{4 a^{2} d^{2} q^{d}}{\left(1-q^{d}\right)^{2}} \\
& +\frac{2 a d^{3} q^{d}}{\left(1-q^{d}\right)^{2}}\left(1-q^{d}\right)+\frac{4 a d^{3} q^{2 d}}{\left(1-q^{d}\right)^{3}}\left(1-q^{d}\right) \\
& +\frac{d^{4} q^{d}}{\left(1-q^{d}\right)^{4}}\left\{\left(1-q^{d}\right)^{3}+10 q^{d}\left(1-q^{d}\right)^{2}+18 q^{2 d}\left(1-q^{d}\right)+9 q^{3 d}\right\} \\
& =>\mu_{4}=\frac{a^{4} d q^{d}}{\left(1-q^{d}\right)}-\frac{4 a^{3} d q^{d}}{\left(1-q^{d}\right)}-\frac{4 d^{2} q^{2 d}}{\left(1-q^{d}\right)^{2}}+\frac{3 a^{3} d}{\left(1-q^{d}\right)}-2 a^{2} d^{2}+a d^{3}+\frac{4 a^{2} d^{2} q^{d}}{\left(1-q^{d}\right)^{2}}+\frac{2 a d^{3} q^{d}}{\left(1-q^{d}\right)}+\frac{4 a d^{3} q^{2 d}}{\left(1-q^{d}\right)^{2}} \\
& +\frac{d^{4} q^{d}}{\left(1-q^{d}\right)^{4}}\left(1-3 q^{d}+3 q^{2 d}-q^{3 d}+10 q^{d}-20 q^{2 d}+10 q^{3 d}+18 q^{2 d}-18 q^{3 d}+9 q^{3 d}\right) \\
& =>\mu_{4}=\frac{a^{4} d q^{d}}{\left(1-q^{d}\right)}-\frac{4 a^{3} d q^{d}}{\left(1-q^{d}\right)}-\frac{4 d^{2} q^{2 d}}{\left(1-q^{d}\right)^{2}}+\frac{3 a^{3} d}{\left(1-q^{d}\right)}-\frac{2 a^{2} d^{2}}{\left(1-q^{d}\right)^{2}}\left(1-2 q^{d}+q^{2 d}-2 q^{d}\right) \\
& +\frac{a d^{3}}{\left(1-q^{d}\right)^{2}}\left(1-2 q^{d}+q^{2 d}+2 q^{d}-2 q^{2 d}+4 q^{d}\right)+\frac{d^{4} q^{d}}{\left(1-q^{d}\right)^{4}}\left(1+7 q^{d}+q^{2 d}\right) \\
& =>\mu_{4}=\frac{a^{4} d q^{d}}{\left(1-q^{d}\right)}-\frac{4 d^{2} q^{2 d}}{\left(1-q^{d}\right)^{2}}+\frac{a^{3} d}{\left(1-q^{d}\right)}\left(3-4 q^{d}\right)-\frac{2 a^{2} d^{2}}{\left(1-q^{d}\right)^{2}}\left(1-4 q^{d}+q^{2 d}\right)+\frac{a d^{3}}{\left(1-q^{d}\right)^{2}}\left(1+4 q^{d}-q^{2 d}\right) \\
& +\frac{d^{4} q^{d}}{\left(1-q^{d}\right)^{4}}\left(1+7 q^{d}+q^{2 d}\right)
\end{aligned}
$$

